\title{
Bioefficacy of insecticides and bio-pesticides against yellow stem borer, Scirpophaga incertulus (walk.) and their effect on spiders in rice crop
}

\author{
P. Singh ${ }^{1}$, Rajendra Singh ${ }^{1}$, S. S. Dhaka ${ }^{2}$, Deepak Kumar ${ }^{1}$, Hitendra Kumar ${ }^{3}$ and \\ Neelam Kumari ${ }^{4}$ \\ ${ }^{1}$ Department of Entomology, ${ }^{2}$ K.V.K. Pilibhit, SVPUAT, Meerut.-250110, India \\ ${ }^{3}$ Department of Entomology, SGRR (PG) College, Deharadun (Uttrakhand) \\ ${ }^{4}$ Department of Entomology, R.B. (P.G.) College, Agra.
}

\begin{abstract}
All the treatments tested for the management of yellow stem borer, Scirpophaga incertulus (Walk.) had the comparable lower number of dead hearts and white ears than un-treated control. Fipronil $5 \mathrm{SC}$ was best with final infestation of $1.9 \%$ and $45.83 \mathrm{q} / \mathrm{ha}$ yield followed by cartap hydrochloride $50 \mathrm{SP}$, triazophos $40 \mathrm{EC}$, cartap hydrochloride 4G, lambda cyhalothrin 5 EC, endosulfan 35 EC, Bt and neemarin which gave 44.96, 43.37, 42.49, 40.13, 39.85, 39.07 and $38.78 \mathrm{q} / \mathrm{ha}$ yield, respectively. Maximum numbers of predatory spiders were recorded in control plot and it was 3.26 / hill. Among the different treatments the maximum number of spiders was found in cartap hydrochloride $4 \mathrm{G}$ followed by fipronil $5 \mathrm{SC}$ as 1.66 and 1.53 / hill, respectively.
\end{abstract}

Key words: Fipronil, Scirpophaga incertulas, predators, Oryza sativa L.

Paper Cited: Singh, P., Singh, R., Dhaka, S.S., Kumar, D., Kumar, H. and Kumari, N. (2015). Bioefficacy of insecticides and bio-pesticides against yellow stem borer, Scirpophaga incertulus (walk.) and their effect on spiders in rice crop. South Asian J. Food Technol. Environ. 1(2): 179-183.

Rice (Oryza sativa L.) is one of the most important cereal crops of India and is a staple food of more than $65 \%$ of its population. The rice crop is subjected to sustain a considerable damage by a number of insect pests, among them yellow stem borer, Scirpophaga incerulas (Walk.) is the principle devastators, which is responsible for economic crop losses under local conditions (Kumar et al., 2012). This insect attacks the crop from the seedling stage to the harvesting stage and thus causes complete loss of affected tillers. Dead hearts are produced when the insect attacks at vegetative stage while white heads occur when the stem borer attack at time of heading. Chemical insecticides are still effective method to control insect pests particularly in the rice crop. The indiscriminate use of chemical insecticides can be environmentally disruptive and can result in the accumulation of residues in the harvested produce. Dodan and Lal (1999); Kaul and Sharma (1999) and Rath (1999 \& 2001). The use of conventional insecticides causes sudden decrease in the number of natural enemies also. Keeping in view the importance of rice crop, the present study was undertaken and results thus obtained are presented herein.

\section{Material and Method}

The field trial was conducted 2010 at Crop Research Centre of the S.V.P.U.A. \&T., Meerut, laid in R.B.D. with nine treatments and 3 replications. The treatments were T1, triazophos 40 EC @ 0.75 lit./ha,
T2, cartap hydrochloride 4G @ 18.75 kg/ha, T3, lambda-cyhalothrin 5 EC @ 0.625 lit/ha, T4, fipronil 5 EC @ 18.75 kg/ha, T5, endosulfan 35 EC @ 1.5 lit/ha., T6, cartap hydrochloride 50SP @ 1.0 kg/ha., T7, neemarin @ 1.5 lit./ha., T8, Bacillus thuringiensis var, kurstaki@1.5 kg/ha. and T9, untreated control. All the treatments were applied twice, first at the 50 days and second at 75 days after transplanting with hand operated knap-sack sprayer, only water was sprayed in untreated control plot, the spray volume used for foliar application of insecticide was 600 litrelha. During spraying due care was taken to prevent insecticides drift and granular insecticide was applied after mixing in $10 \mathrm{Kg}$ sand/acre. The observations on dead hearts and white ear formation due to yellow stem borer were taken on five randomly selected hills / plot, before 1 day and after 3, 7, 14 and 20 one days of application. The percentage of dead hearts / white ears was worked out.

Grain yield / plot was also recorded at harvest and it was converted in to quintal / ha. for analysis and comparison. The economics of each treatment was also worked out on the basis of cost benefit ratio.

\section{Results and Discussion}

All the treatments were significantly effective in reducing the infestation of rice yellow stem borer (YSB) and thus, reducing the formation of dead heart and white ear significantly as compared to the control (Table 1). 
Table 1. Bio-efficacy of insecticides and bio-pesticides against Scirpophaga incertulas in rice.

\begin{tabular}{|c|c|c|c|c|c|c|c|c|c|c|}
\hline \multirow{3}{*}{ Treatments } & \multirow{3}{*}{ Dose / ha. } & \multicolumn{9}{|c|}{ \% dead hearts / white ears } \\
\hline & & \multirow{2}{*}{$\begin{array}{l}\text { before } \\
\text { spray }\end{array}$} & \multicolumn{4}{|c|}{ First Application } & \multicolumn{4}{|c|}{ Second Application } \\
\hline & & & 3 DAA & 7 DAA & 14 DAA & 21 DAA & 3 DAA & 7 DAA & 14 DAA & 21 DAA \\
\hline T1. Triazophos 40 EC & 0.75 lit. & $\begin{array}{c}4.96 \\
(12.87) \\
\end{array}$ & $\begin{array}{c}2.43 \\
(8.62) \\
\end{array}$ & $\begin{array}{c}2.96 \\
(9.91) \\
\end{array}$ & $\begin{array}{c}3.60 \\
(9.96) \\
\end{array}$ & $\begin{array}{c}4.23 \\
(11.76) \\
\end{array}$ & $\begin{array}{c}1.96 \\
(7.93) \\
\end{array}$ & $\begin{array}{c}2.46 \\
(8.93) \\
\end{array}$ & $\begin{array}{c}2.96 \\
(9.91) \\
\end{array}$ & $\begin{array}{c}2.99 \\
(9.70) \\
\end{array}$ \\
\hline $\begin{array}{l}\text { T2. Cartap } \\
\text { hydrochloride } 4 \mathrm{G}\end{array}$ & $18.75 \mathrm{~kg}$ & $\begin{array}{c}4.70 \\
(12.31)\end{array}$ & $\begin{array}{c}2.80 \\
(9.42)\end{array}$ & $\begin{array}{c}3.26 \\
(10.37)\end{array}$ & $\begin{array}{c}3.96 \\
(11.28)\end{array}$ & $\begin{array}{c}4.43 \\
(12.07)\end{array}$ & $\begin{array}{c}2.36 \\
(8.74)\end{array}$ & $\begin{array}{c}2.83 \\
(9.47)\end{array}$ & $\begin{array}{c}3.73 \\
(11.13)\end{array}$ & $\begin{array}{c}4.10 \\
(11.67)\end{array}$ \\
\hline $\begin{array}{l}\text { T3. Lambda-cyhalothrin } \\
5 \text { EC }\end{array}$ & 0.625 lit. & $\begin{array}{c}5.16 \\
(12.93)\end{array}$ & $\begin{array}{c}3.10 \\
(9.92)\end{array}$ & $\begin{array}{c}3.60 \\
(10.56)\end{array}$ & $\begin{array}{c}4.06 \\
(11.17)\end{array}$ & $\begin{array}{c}4.63 \\
(12.23)\end{array}$ & $\begin{array}{c}2.06 \\
(8.17)\end{array}$ & $\begin{array}{c}2.60 \\
(8.97) \\
\end{array}$ & $\begin{array}{c}2.93 \\
(9.64) \\
\end{array}$ & $\begin{array}{c}3.13 \\
(10.04)\end{array}$ \\
\hline T4. Fipronil 5 SC & 0.5 lit. & $\begin{array}{c}5.03 \\
(12.91)\end{array}$ & $\begin{array}{c}1.73 \\
(7.54) \\
\end{array}$ & $\begin{array}{c}2.40 \\
(8.40)\end{array}$ & $\begin{array}{c}2.40 \\
(8.90)\end{array}$ & $\begin{array}{c}3.43 \\
(10.65)\end{array}$ & $\begin{array}{c}0.93 \\
(4.52) \\
\end{array}$ & $\begin{array}{c}1.03 \\
(4.76)\end{array}$ & $\begin{array}{c}1.46 \\
(6.94)\end{array}$ & $\begin{array}{c}1.93 \\
(7.92)\end{array}$ \\
\hline T5. Endosulfan 35 EC & 1.5 lit. & $\begin{array}{c}5.23 \\
(13.13)\end{array}$ & $\begin{array}{c}3.30 \\
(10.09)\end{array}$ & $\begin{array}{c}3.76 \\
(11.03)\end{array}$ & $\begin{array}{c}4.10 \\
(11.43)\end{array}$ & $\begin{array}{c}4.70 \\
(11.98)\end{array}$ & $\begin{array}{c}3.26 \\
(10.37)\end{array}$ & $\begin{array}{c}3.76 \\
(11.15)\end{array}$ & $\begin{array}{c}4.26 \\
(11.91)\end{array}$ & $\begin{array}{c}4.36 \\
(12.05) \\
\end{array}$ \\
\hline $\begin{array}{l}\text { T6. Cartap } \\
\text { hydrochloride 50SP }\end{array}$ & $1.0 \mathrm{~kg}$ & $\begin{array}{c}5.53 \\
(13.49) \\
\end{array}$ & $\begin{array}{c}2.30 \\
(8.05) \\
\end{array}$ & $\begin{array}{c}2.90 \\
(9.60) \\
\end{array}$ & $\begin{array}{c}3.13 \\
(9.79) \\
\end{array}$ & $\begin{array}{c}3.90 \\
(11.19) \\
\end{array}$ & $\begin{array}{c}1.63 \\
(7.33) \\
\end{array}$ & $\begin{array}{c}1.93 \\
(7.89) \\
\end{array}$ & $\begin{array}{c}2.43 \\
(8.88) \\
\end{array}$ & $\begin{array}{c}2.96 \\
(9.60) \\
\end{array}$ \\
\hline T7. B.t. & $1.5 \mathrm{~kg}$ & $\begin{array}{c}5.63 \\
(13.53)\end{array}$ & $\begin{array}{c}4.03 \\
(11.54)\end{array}$ & $\begin{array}{c}4.60 \\
(12.29)\end{array}$ & $\begin{array}{c}5.00 \\
(12.80)\end{array}$ & $\begin{array}{c}5.93 \\
(12.08)\end{array}$ & $\begin{array}{c}4.13 \\
(11.68)\end{array}$ & $\begin{array}{c}4.66 \\
(12.35)\end{array}$ & $\begin{array}{c}5.13 \\
(12.96)\end{array}$ & $\begin{array}{c}5.60 \\
(13.68)\end{array}$ \\
\hline T8. Neemarin 1500 ppm & 3.0 lit & $\begin{array}{c}6.30 \\
(14.42) \\
\end{array}$ & $\begin{array}{c}4.96 \\
(12.85) \\
\end{array}$ & $\begin{array}{c}5.26 \\
(13.23) \\
\end{array}$ & $\begin{array}{c}5.60 \\
(13.67) \\
\end{array}$ & $\begin{array}{c}6.10 \\
(14.28) \\
\end{array}$ & $\begin{array}{c}5.73 \\
(13.79) \\
\end{array}$ & $\begin{array}{c}5.93 \\
(13.93) \\
\end{array}$ & $\begin{array}{c}6.30 \\
(14.43) \\
\end{array}$ & $\begin{array}{c}6.90 \\
(15.17) \\
\end{array}$ \\
\hline T9. Control & & $\begin{array}{c}4.83 \\
(12.55) \\
\end{array}$ & $\begin{array}{c}6.66 \\
(14.91) \\
\end{array}$ & $\begin{array}{c}7.53 \\
(15.92) \\
\end{array}$ & $\begin{array}{c}8.26 \\
(16.70) \\
\end{array}$ & $\begin{array}{c}8.80 \\
(17.24) \\
\end{array}$ & $\begin{array}{c}8.80 \\
(17.23) \\
\end{array}$ & $\begin{array}{c}8.56 \\
(16.99) \\
\end{array}$ & $\begin{array}{c}9.06 \\
(17.51) \\
\end{array}$ & $\begin{array}{c}9.33 \\
(17.78) \\
\end{array}$ \\
\hline SEm \pm & & NS & 1.27 & 1.13 & 1.21 & 1.05 & 1.03 & 1.32 & 0.85 & 0.87 \\
\hline $\mathrm{CD}(\mathrm{P}=0.05)$ & & & 3.85 & 3.45 & 3.66 & 3.17 & 3.13 & 3.99 & 2.58 & 2.64 \\
\hline
\end{tabular}

(Figures in parentheses are angular transformed values; DAA = Days after application) 
Table 2. Effect of different treatments on spider's population.

\begin{tabular}{|c|c|c|c|c|c|c|c|c|c|c|}
\hline \multirow{3}{*}{ Treatments } & \multirow{3}{*}{ Dose/ha } & \multicolumn{9}{|c|}{ Number of spiders/hill } \\
\hline & & \multirow{2}{*}{$\begin{array}{l}\text { before } \\
\text { spray }\end{array}$} & \multicolumn{4}{|c|}{ First Application } & \multicolumn{4}{|c|}{ Second Application } \\
\hline & & & 3 DAA & 7 DAA & 14 DAA & 21 DAA & 3 DAA & 7 DAA & 14 DAA & 21 DAA \\
\hline T1. Triazophos 40 EC & 0.75 lit. & $\begin{array}{c}1.33 \\
(1.53)\end{array}$ & $\begin{array}{c}0.60 \\
(1.26)\end{array}$ & $\begin{array}{c}0.66 \\
(1.29)\end{array}$ & $\begin{array}{c}0.80 \\
(1.34)\end{array}$ & $\begin{array}{c}1.00 \\
(1.41)\end{array}$ & $\begin{array}{c}0.60 \\
(1.26)\end{array}$ & $\begin{array}{c}0.80 \\
(1.34)\end{array}$ & $\begin{array}{c}0.86 \\
(1.37)\end{array}$ & $\begin{array}{c}1.0 \\
(1.41)\end{array}$ \\
\hline T2. Cartap hydrochloride $4 \mathrm{G}$ & $18.75 \mathrm{~kg}$ & $\begin{array}{c}1.20 \\
(1.48)\end{array}$ & $\begin{array}{c}1.06 \\
(1.43)\end{array}$ & $\begin{array}{c}1.13 \\
(1.46)\end{array}$ & $\begin{array}{c}1.66 \\
(1.63)\end{array}$ & $\begin{array}{c}1.73 \\
(1.64)\end{array}$ & $\begin{array}{c}1.46 \\
(1.57)\end{array}$ & $\begin{array}{c}1.40 \\
(1.55)\end{array}$ & $\begin{array}{c}1.60 \\
(1.61)\end{array}$ & $\begin{array}{c}1.66 \\
(1.63)\end{array}$ \\
\hline T3. Lambda-cyhalothrin 5 EC & 0.625 lit. & $\begin{array}{c}1.13 \\
(1.46) \\
\end{array}$ & $\begin{array}{c}0.73 \\
(1.31) \\
\end{array}$ & $\begin{array}{c}0.80 \\
(1.34) \\
\end{array}$ & $\begin{array}{c}0.86 \\
(1.36) \\
\end{array}$ & $\begin{array}{c}1.06 \\
(1.41) \\
\end{array}$ & $\begin{array}{c}0.73 \\
(1.31) \\
\end{array}$ & $\begin{array}{c}0.80 \\
(1.34) \\
\end{array}$ & $\begin{array}{c}0.93 \\
(1.39) \\
\end{array}$ & $\begin{array}{c}1.06 \\
(1.44) \\
\end{array}$ \\
\hline T4. Fipronil 5 SC & 0.5 lit. & $\begin{array}{c}1.06 \\
(1.44) \\
\end{array}$ & $\begin{array}{c}1.00 \\
(1.41) \\
\end{array}$ & $\begin{array}{c}0.93 \\
(1.39) \\
\end{array}$ & $\begin{array}{c}1.13 \\
(1.46) \\
\end{array}$ & $\begin{array}{c}1.66 \\
(1.63) \\
\end{array}$ & $\begin{array}{c}1.20 \\
(1.48)\end{array}$ & $\begin{array}{c}1.33 \\
(1.52) \\
\end{array}$ & $\begin{array}{c}1.40 \\
(1.54) \\
\end{array}$ & $\begin{array}{c}1.53 \\
(1.59)\end{array}$ \\
\hline T5. Endosulfan 35 EC & 1.5 lit. & $\begin{array}{c}1.46 \\
(1.56) \\
\end{array}$ & $\begin{array}{c}0.53 \\
(1.23) \\
\end{array}$ & $\begin{array}{c}0.60 \\
(1.26) \\
\end{array}$ & $\begin{array}{c}0.74 \\
(1.31) \\
\end{array}$ & $\begin{array}{c}0.93 \\
(1.39) \\
\end{array}$ & $\begin{array}{c}0.53 \\
(1.24) \\
\end{array}$ & $\begin{array}{c}0.73 \\
(1.31) \\
\end{array}$ & $\begin{array}{c}0.86 \\
(1.37) \\
\end{array}$ & $\begin{array}{c}0.93 \\
(1.39) \\
\end{array}$ \\
\hline T6. Cartap hydrochloride 50SP & $1.0 \mathrm{~kg}$ & $\begin{array}{c}1.00 \\
(1.41) \\
\end{array}$ & $\begin{array}{c}0.80 \\
(1.34) \\
\end{array}$ & $\begin{array}{c}0.86 \\
(1.36) \\
\end{array}$ & $\begin{array}{c}0.87 \\
(1.39) \\
\end{array}$ & $\begin{array}{c}1.13 \\
(1.46) \\
\end{array}$ & $\begin{array}{c}0.86 \\
(1.37) \\
\end{array}$ & $\begin{array}{c}0.93 \\
(1.39) \\
\end{array}$ & $\begin{array}{c}1.06 \\
(1.43) \\
\end{array}$ & $\begin{array}{c}1.20 \\
(1.48) \\
\end{array}$ \\
\hline T7. B. t. & $1.5 \mathrm{~kg}$ & $\begin{array}{c}1.46 \\
(1.57) \\
\end{array}$ & $\begin{array}{c}1.26 \\
(1.50) \\
\end{array}$ & $\begin{array}{c}1.40 \\
(1.55)\end{array}$ & $\begin{array}{c}1.60 \\
(1.61) \\
\end{array}$ & $\begin{array}{c}2.26 \\
(1.79) \\
\end{array}$ & $\begin{array}{c}2.00 \\
(1.73) \\
\end{array}$ & $\begin{array}{c}1.86 \\
(1.69) \\
\end{array}$ & $\begin{array}{c}1.93 \\
(1.71) \\
\end{array}$ & $\begin{array}{c}2.06 \\
(1.75) \\
\end{array}$ \\
\hline T8. Neemarin 1500 ppm & 3.0 lit & $\begin{array}{c}1.26 \\
(1.50) \\
\end{array}$ & $\begin{array}{c}1.33 \\
(1.53) \\
\end{array}$ & $\begin{array}{c}1.46 \\
(1.57) \\
\end{array}$ & $\begin{array}{c}1.66 \\
(1.63) \\
\end{array}$ & $\begin{array}{c}2.53 \\
(1.88) \\
\end{array}$ & $\begin{array}{c}2.26 \\
(1.81) \\
\end{array}$ & $\begin{array}{c}2.53 \\
(1.88) \\
\end{array}$ & $\begin{array}{c}2.60 \\
(1.89) \\
\end{array}$ & $\begin{array}{c}2.66 \\
(1.91) \\
\end{array}$ \\
\hline T9. Control & & $\begin{array}{c}1.20 \\
(1.47)\end{array}$ & $\begin{array}{c}1.93 \\
(1.71)\end{array}$ & $\begin{array}{c}2.06 \\
(1.75)\end{array}$ & $\begin{array}{c}2.73 \\
(1.93)\end{array}$ & $\begin{array}{c}2.93 \\
(1.98)\end{array}$ & $\begin{array}{c}3.13 \\
(2.03)\end{array}$ & $\begin{array}{c}3.40 \\
(2.09)\end{array}$ & $\begin{array}{c}3.33 \\
(2.08)\end{array}$ & $\begin{array}{c}3.26 \\
(2.06)\end{array}$ \\
\hline SEm \pm & & NS & 0.04 & 0.05 & 0.06 & 0.07 & 0.05 & 0.05 & 0.06 & 0.06 \\
\hline $\mathrm{CD}(\mathrm{P}=0.05)$ & & & 0.13 & 0.14 & 0.14 & 0.19 & 0.15 & 0.16 & 0.17 & 0.18 \\
\hline
\end{tabular}

(Figures in parentheses are square root transformed values; DAA = Days after application) 
Table 3. Economics of different treatments in management of Scirpophaga incertulas

\begin{tabular}{|l|c|c|c|c|c|c|c|}
\hline Treatments & $\begin{array}{c}\text { Total cost of } \\
\text { treatment } \\
\text { application } \\
\text { (Rs./ha.) }\end{array}$ & $\begin{array}{c}\text { Yield } \\
(\mathbf{q} / \mathbf{h a .})\end{array}$ & $\begin{array}{c}\text { Yield } \\
\text { obtained } \\
\text { over } \\
\text { (ontrol } \\
\text { (q/ha.) }\end{array}$ & $\begin{array}{c}\text { Value of } \\
\text { increase } \\
\text { d } \\
\text { yield } \\
\text { (Rs/ha.) }\end{array}$ & $\begin{array}{c}\text { Gross } \\
\text { income } \\
\text { (Rs/ha.) }\end{array}$ & $\begin{array}{c}\text { Net } \\
\text { income } \\
\text { (Rs/ha.) }\end{array}$ & $\begin{array}{c}\text { Cost: } \\
\text { Benefit } \\
\text { Ratio }\end{array}$ \\
\hline $\begin{array}{l}\text { T1. Triazophos 40 } \\
\text { EC }\end{array}$ & 1555.00 & 43.37 & 7.98 & 15162.00 & 82403.00 & 80848.00 & $1: 9.75$ \\
\hline $\begin{array}{l}\text { T2. Cartap } \\
\text { hydrochloride 4G }\end{array}$ & 2717.00 & 42.49 & 7.10 & 13490.00 & 80731.00 & 78014.00 & $1: 4.96$ \\
\hline $\begin{array}{l}\text { T3. Lambda- } \\
\text { cyhalothrin 5 EC }\end{array}$ & 1030.000 & 40.13 & 4.74 & 9006.00 & 76247.00 & 75217.00 & $1: 8.74$ \\
\hline T4. Fipronil 5 SC & 1380.00 & 45.83 & 10.44 & 19836.00 & 87077.00 & 85897.00 & $1: 14.4$ \\
\hline $\begin{array}{l}\text { T5. Endosulfan 35 } \\
\text { EC }\end{array}$ & 1030.00 & 39.85 & 4.46 & 8474.00 & 75715.00 & 74835.00 & $1: 8.23$ \\
\hline $\begin{array}{l}\text { T6. Cartap } \\
\text { hydrochloride 50SP }\end{array}$ & 1880.00 & 44.96 & 9.57 & 18183.00 & 85424.00 & 83544.00 & $1: 9.67$ \\
\hline T7. B.t & 2562.00 & 39.07 & 3.68 & 6992.00 & 74233.00 & 71818.00 & $1: 2.73$ \\
\hline $\begin{array}{l}\text { T8. Neemarin 1500 } \\
\text { ppm }\end{array}$ & 25549.00 & 38.78 & 3.39 & 6441.00 & 73682.00 & 48133.00 & $1: 0.25$ \\
\hline T9. Control & - & 35.39 & - & - & - & - & - \\
\hline
\end{tabular}

Labour charges $=$ Rs. 100/day; Rental value of sprayer $=$ Rs. 20.00/day; Sale price of produce $=$ Rs. 1900 /quintal

In treated plots, the yellow stem borer infestation recorded as dead hearts and white ears ranged from 1.93 to 6.90 as against $9.33 \%$ in control, after 21 days of second spray. Fipronil 5 SC @ 0.5 lit. / ha. was found most effective against YSB followed by cartap hydrochloride $50 \mathrm{SP} @ 1.0 \mathrm{~kg}$. / ha. and triazophos 40 EC @ 0.75 lit/ha. Although, statistical analyses showed non-significant difference among various treatments but control had significantly high $(9.33 \%)$ dead hearts / white ears than in treated plots, after 21 days of second spray. It gave clear message that chemical insecticides including bio-pesticides suppressed the population of yellow stem borer in rice.

The present finding in agreement with the findings of Dhaka et al., (2011), who reported fipronil $5 \mathrm{SC}$ as the best treatment in reducing the infestation of insect pests in rice, Sachan et al., (2006) reported cartap hydrochloride $50 \mathrm{SP}$ as the second best treatment in managing the stem borer in rice, Prasad et al., (2005) found fipronil 0.3 GR at $0.075 \mathrm{~kg}$ a.i./ha most effective in controlling yellow stem borer infestation. As regards the bio-pesticides, the neemarin was found better than the Bt though on par statistically. The percentage of dead hearts / white ears after 21 days of second spray was 5.6 and 6.9 with neemarin and $\mathrm{Bt}$, respectively. These results are in conformity with the findings of Dhaka et al., (2011); Kumar et al., (2012) and Prasad et al., (2004) who found neem products to control yellow rice stem borer. These findings do not corroborates with the findings of Singh et al., (2008) who reported that Bt as second best treatment in controlling the yellow stem borer among all the treatments.

The results on the availability of spiders in different treatments (Table 2) showed that highest number of spiders, was found in the un-treated control (3.26 / hills after 21 days of second spray), which was higher than the number of spiders recorded in other treatments. The least number of the spiders were recorded in the treatment of endosulfan 35 EC. The results indicated that $\mathrm{Bt}$, neemarin, fipronil $5 \mathrm{SC}$, cartap hydrochloride $50 \mathrm{SP}$, and cartap hydrochloride $4 \mathrm{G}$ had no adverse effect on predators. The present studies are in conformity with several workers viz. Kadam et al., (2005) who reported the maximum number of spider populations in the plot treated with neem oil spray and NSKE and also with Dhaka et al., (2011) who also reported fipronil 0.3 GR and cartap hydrochloride $4 \mathrm{G}$ a bit safer for the spiders.

\section{Conclusion}

The plots treated with fipronil 5SC (45.83 q/ha), cartap hydrochloride 50 SP (44.96 q./ha) and triazophos 40 EC (43.37 q/ha) gave higher yield among the treatments and the yield in all treatments was found significantly higher than untreated control ( $35.39 \mathrm{q} / \mathrm{ha})$. The findings are in confirmation with Nayak et al., (2000). In terms of economics the highest net return was also obtained with fipronil 5 SC followed by cartap hydrochloride $50 \mathrm{SP}$ and triazophos 40 EC. The highest cost: benefit ratio was 
also found with fipronil 5 SC (14.4) followed by triazophos $40 \mathrm{EC}(9.75)$ and cartap hydrochloride 50 SP (9.67). Similar results on yield component was reported by Dhaka et al., (2011). The results reveal that since the plots treated with fipronil $5 \mathrm{SC}$ had the lowest dead hearts / white ears percentage and produced maximum paddy yield than other treatments and more number of predators were also found in rice field.

\section{References}

1. Dhaka, S.S., Prajapati C.R., Singh D V. and Singh, R. (2011). Field evaluation of insecticides and biopesticides against rice leaf folder, Cnaphalocrosis medinalis. Ann Plant Protect Sci. 19(2): 324-326.

2. Dodan, D.S. and Lal, R. (1999). Integrated management of neck blast and stem borer in scented rice. Haryana Agric Univ J Res. 29 (1/2): 47-49.

3. Kadam, J.R., Bhosle, U.D., Chavan, A.P. and Mhase, B.M. (2005). Bioefficacy of insecticides sequences against pests of brinjal and their impact on natural enemies. Ann Plant Protect Sci. 13(2): 278-282.

4. Kaul, B.K. and Sharma, P.K. (1999). Efficacy of neem based insecticides against the major insect pests of rice in the hills of Himachal Pradesh (India). J Entomol Res. 23 (4): $377-$ 379.

5. Kumar, Amit; Lal, M.N., Singh A.K. and Prasad, C.S. (2012). Eco-friendly management of Scirpophaga incertulus (Walk.) Ann Plant Protect Sci. 20(2): 211-212.
6. Nayak, S.K., Panda, S.K. and Behera, U.K. (2000). Impact of varietal resistance and insecticides on rice stem borer and gall midge. Ann Plant Protect Sci. 8 (2): 140-144.

7. Prasad, S.S. Gupta, P.K. Kanaujia, B.L. (2005). Efficacy and economics of promising granular insecticides against yellow stem borer on semi-deep water rice. Ann Plant Protect Sci. 13 (2): 365-368.

8. Prasad, S.S., Gupta, P.K., Singh, R.B. and Kanaujia, B.L. (2004). Evaluation of neem products tested against yellow rice stem borer, Scirpopapha incertulas on deep water rice. Ann Plant Protect Sci. 11(2): 426-428.

9. Rath, P.C. (1999). Evaluation of some Bacillus thuringiensis and neem formulations against yellow stem borer of rice under rainfed lowlands. Oryza. 36: 398-399.

10. Rath, P.C. (2001). Efficacy of insecticides, neem and $\mathrm{Bt}$ formulation against stem borer on rice yield in West Bengal. J Appl Zool Res. 12: 191-93.

11. Sachan, S.K., Singh, D.V. and Chaudhary, A.S. (2006). Field evaluation of insecticides against rice stem borer and leaf folder. Ann Plant Protect Sci. 14(2). 462-464.

12. Singh, S., Prasad, C.S., Nath, L. and Tiwari, G.N. (2008). Eco-friendly Management of Scirpophaga incertulas (Walk.) and Cnaphalocrocis medinalis (Guen.) in Basmati Rice. Ann Plant Protect Sci. 16(1): 11-16. 